\title{
Fifteen months follow-up of a hopeless tooth treated with two-step procedure involving intentional replantation and free gingival graft
}

\author{
Huseyin Gencay Keceli ${ }^{1}$, Meltem Karsiyaka Hendek ${ }^{2}$
}

Correspondence: Dr. Huseyin Gencay Keceli

Email: monsieur_gencay@yahoo.com

\author{
'Department of Periodontology, Faculty of Dentistry, \\ Hacettepe University, Ankara, Turkiye, \\ 'Department of Periodontology, Faculty of Dentistry, \\ Kirikkale University, Kirikkale, Turkiye
}

\section{ABSTRACT}

The aim of the report is to document 15-month results of mucogingival surgery applied following intentional replantation (IntR). A mandibular left lateral incisor with severe periodontitis and malposition was replanted and splinted following root canal treatment. Three months after IntR, a free gingival graft (FGG) was placed. Fifteen months follow-up of the case demonstrated significant improvement in periodontal healing parameters and significant bone fill was detected around the root apex. As a conclusion, IntR may be speculated as a convenient alternative to keep the periodontally hopeless teeth and supportive approaches such as FGGs may improve the outcomes following IntR.

Key words: Free gingival graft, intentional replantation, mucogingival problem, periodontally hopeless tooth

\section{INTRODUCTION}

Intentional replantation ( $\mathrm{IntR}$ ) is described as the intervention of purposely removing a tooth and following some extra-oral procedures, replacing it into its socket. ${ }^{[1]}$ Despite increasing tendency to be a favored treatment alternative in cases of periodontally hopeless teeth (PHT), ${ }^{[1-3]}$ enhancement of periodontal tissue support and thus, increasing the lifetime and functional quality of replanted teeth is still one of the main concerns. Supportive treatments have been suggested to improve results obtained with IntR. ${ }^{[2-5]}$ Despite the contribution of these to periodontal health, only periodontal pockets and inflammation were evaluated, and no detailed information was given about periodontal and mucogingival state.

Because of thin tissue phenotype, PHT in mandibular anterior area may frequently subject to gingival recession in case of periodontal destruction. Therefore, mucogingival problem should be potentially considered in performing IntR. Although reports involving IntR point out outcomes about survival and reduction of inflammation around treated teeth, there is limited data indicating the importance of mucogingival state. ${ }^{[3]}$ In addition, no report represented treatment of PHT with a two-step procedure involving IntR and free gingival graft (FGG). Therefore, objective of this report is to present 15-month results of treatment including IntR and mucogingival surgery. The report also aims to underline the contribution of supporting approaches to Int $R$ procedure in terms of periodontal tissue support and amount of keratinized gingiva.

\section{CASE REPORT}

A 20-year-old female was referred to Kirikkale University Periodontology Department in June 2011 with mobility and discomfort in mandibular left lateral incisor. Clinically, tooth had malposition, severe

\footnotetext{
How to cite this article: Keceli HG, Hendek MK. Fifteen months follow-up of a hopeless tooth treated with two-step procedure involving intentional replantation and free gingival graft. Eur J Dent 2014;8:559-62.

Copyright @ 2014 Dental Investigations Society. DOI: $10.4103 / 1305-7456.143644$
} 
periodontitis with $3^{\circ}$ mobility, inflammation, and recession [Figure 1]. Baseline measurements included plaque index, ${ }^{[6]}$ gingival index, ${ }^{[7]}$ probing depth (from the gingival margin [GM] to base of pocket), attachment level (from cemento-enamel junction [CEJ] to base of pocket), gingival recession (from CEJ to GM), and keratinized gingiva (from GM to mucogingival junction) [Table 1]. Radiographically, the root had huge radiolucent area demonstrating crater-shaped bony defect starting from the coronal third reaching to apical with completely lost buccal + lingual walls [Figure 2].

After treatment planning, informed consent was signed, and remaining treatments were completed. One week after endodontic therapy, IntR was performed using the technique applied by Demiralp et al. ${ }^{[1]}$ In brief, the tooth was extracted and put on a sterilized moisturized sponge [Figure 3]. After scaling and root planning, $100 \mathrm{mg} / \mathrm{ml}$ tetracycline

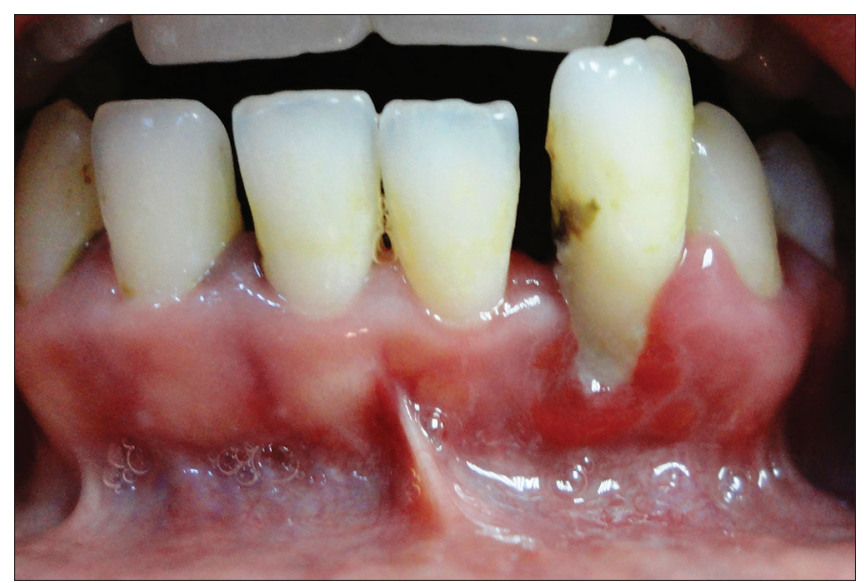

Figure 1: Pretreatment view of the mandibular left lateral incisor demonstrating significant coronal and lateral displacement with hyperemic and edematous gingiva

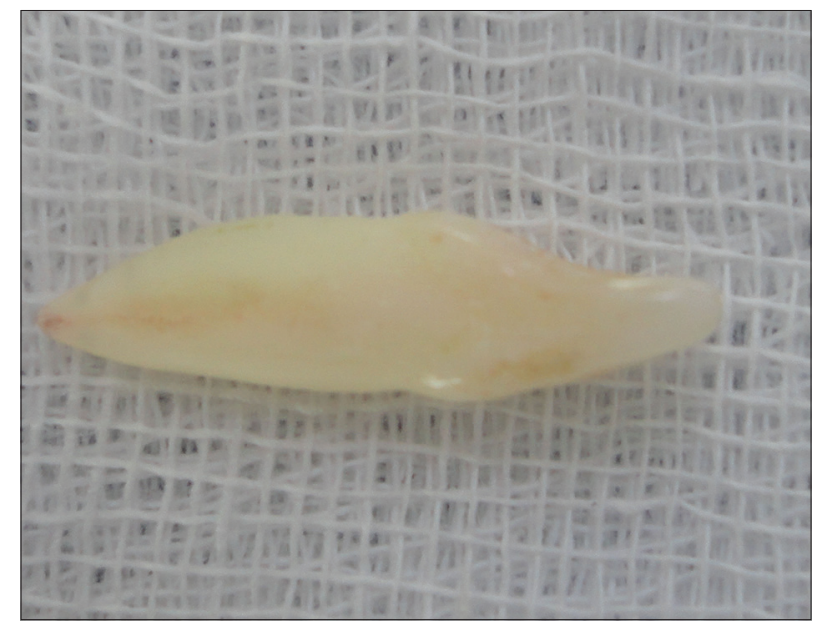

Figure 3: The tooth was gently extracted and the root surface was scaled and root planed
$\mathrm{HCl}$ (Boehringer, Ingelheim am Rhein, Germany) was applied to the root surface for $5 \mathrm{~min}$. The surface was rinsed with sterile distilled water for $1 \mathrm{~min}$, granulation tissue inside the socket was gently debrided and tooth was replaced into the socket. Tooth was repositioned in most possible ideal position to eliminate traumatic occlusion resulted due to pathologic migration. Tooth stabilization was achieved from the incisal third with composite restorative material (3M FiltekTM Z250, 3M Espe, St. Paul, MN, USA). Occlusion and embrasures were checked. After IntR, doxycycline was prescribed for 7 days and oral hygiene activities were continued. Professional cleaning was made at $3^{\text {rd }}$ week, and maintenance visits were scheduled.

Three months after, radiographic view was uneventful, and pocket depths were shallow [Table 1]. However, inflammation and mucogingival stress (no keratinized tissue) was detected [Figure 4]. As the second step, FGG was utilized in accordance with the technique described by Miller ${ }^{[8]}$ [Figure 5]. Sutures were removed 10 days following surgery and toothbrushing was discontinued during this time. Recall visits including professional prophylaxis were

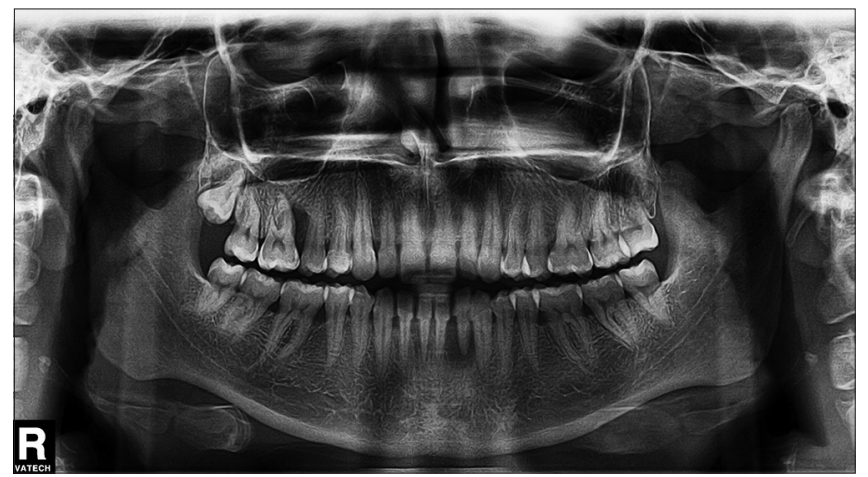

Figure 2: Pretreatment radiographic view. Supporting alveolar bone was completely lost

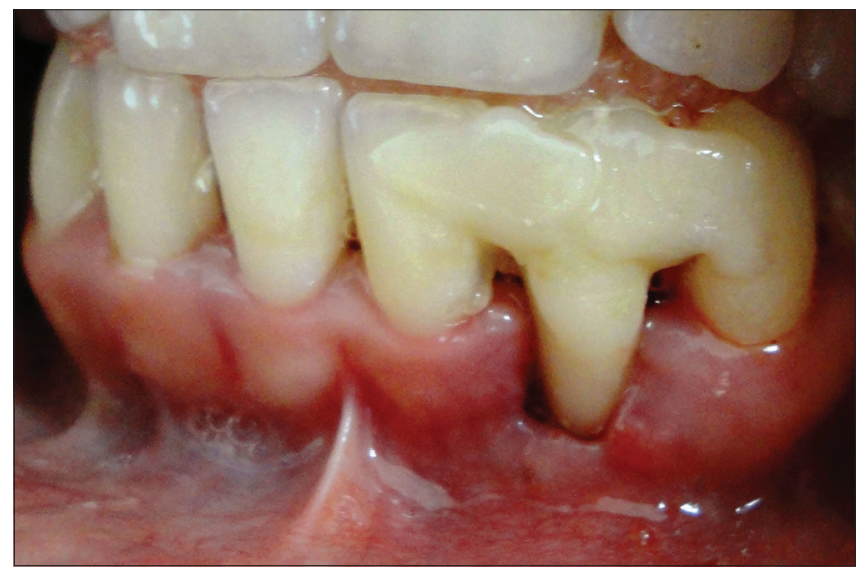

Figure 4: Three months following replantation procedure. Note the lack of keratinized tissue 
arranged. At 15 months, splint was removed, and mobility was reevaluated.

Wilcoxon test was performed to evaluate the time-dependent change of the clinical parameters and significance level was set at $P=0.05$. Compared with baseline, significant improvement was detected at 6 , 9, and 15 months periodontal measurements [Table 1]. Keratinized gingiva width was $8 \mathrm{~mm}$ in follow-up visits. Although gingival recession did not change at 6 months postsurgical visit, a slight decrease was observed in the subsequent follow-up. Gingiva was healthy with its firm view and no bleeding on probing was detected [Figure 6]. Radiographically, the amount of radiolucent area was diminished, and hard tissue formation was detectable around the root apex with no root resorption [Figure 7]. Although slight discoloration occurred in attached gingiva and tooth toward the end of follow-up, patient was satisfied with outcomes of treatment. Following splint removal, $1^{\circ}$ mobility was observed, and control appointments were arranged.

\section{DISCUSSION}

In modern periodontology, growing amount of evidence indicates that several therapeutic modalities can successfully result in keeping PHT in place. Cortellini et al. ${ }^{[9]}$ compared outcomes of periodontal treatment with extraction + prosthetic replacement for PHT and suggested that last step therapeutic procedures should be considered before extraction of PHT. IntR, another last resort therapy,
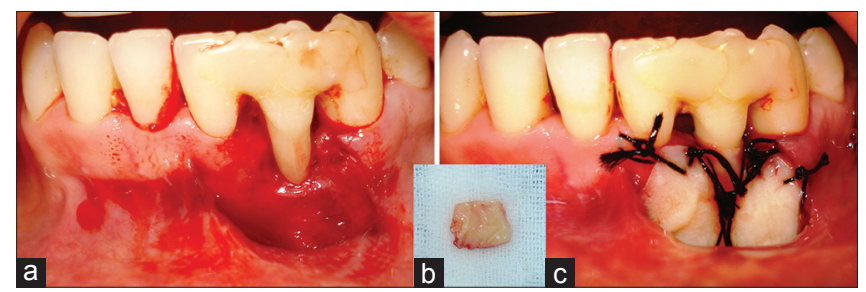

Figure 5: (a) Recipient site prepared for the graft. (b) Free gingival graft tissue. (c) View following graft suturation has demonstrated successful results in the treatment of PHT. ${ }^{[1,2,5]}$ In the present report, significant hard tissue formation was obtained, and replanted tooth was successfully retained during 15 months.

Even though FGG was proposed as supportive in mucogingival problems and prosthodontic and orthodontic treatments, it has not been suggested following IntR yet. In the present case, a mucogingival problem was detected after IntR and it was decided to utilize FGG around the replanted tooth. Following surgery, in addition to the resolution of inflammation, $8 \mathrm{~mm}$ keratinized gingiva and minimal root coverage was obtained. Consequently, it can be recommended that mucogingival problems and supportive treatment alternatives should be considered following IntR procedure in which time the inflammation dissolves and clear decision can be made to perform mucogingival surgery.

Enhancement of periodontal tissue support and thus, increasing the lifetime and functional quality of the replanted teeth is still one of the main concerns. In 2006, Tözüm et al. ${ }^{[3]}$ described use of platelet

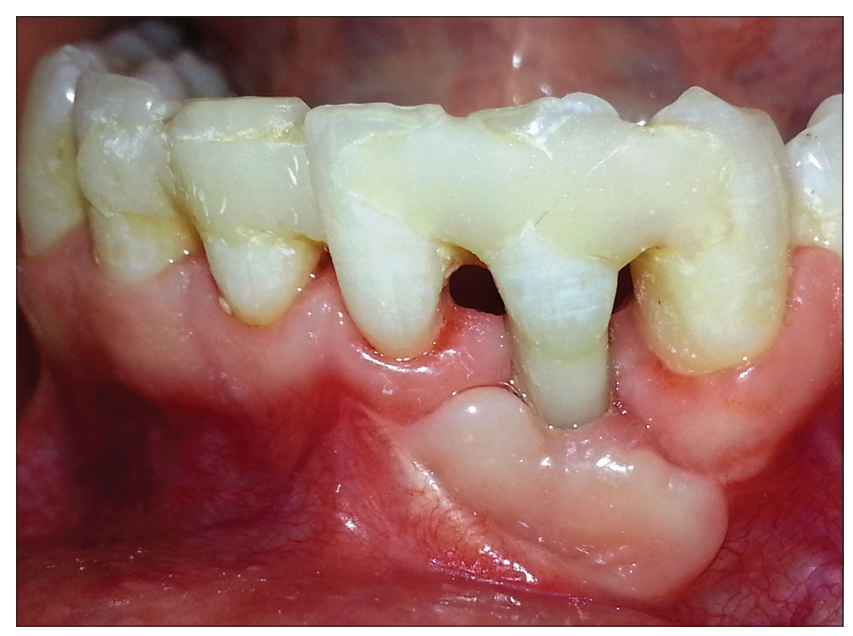

Figure 6: Clinical view at 15 months follow-up. Note that the splint was removed and the gingiva was firm and healthy with a slight color change

\begin{tabular}{|c|c|c|c|c|c|c|c|c|c|c|c|}
\hline & \multirow[t]{2}{*}{ PI } & \multirow[t]{2}{*}{ GI } & \multirow[t]{2}{*}{ Site } & \multicolumn{3}{|c|}{ PD (mm) } & \multicolumn{3}{|c|}{$\mathrm{AL}(\mathrm{mm})$} & \multirow[t]{2}{*}{$\mathrm{KT}(\mathrm{mm})$} & \multirow[t]{2}{*}{$\mathrm{GR}(\mathrm{mm})$} \\
\hline & & & & Mesial & Mid-buccal & Distal & Mesial & Mid-buccal & Distal & & \\
\hline \multirow[t]{2}{*}{ Baseline (before IntR) } & 3 & 2 & Buccal & 11 & 5 & 13 & 16 & 9 & 13 & 1.5 & 4 \\
\hline & & & Lingual & 12 & 7 & 13 & 16 & 11 & 13 & & 4 \\
\hline \multirow[t]{2}{*}{3 months (before FGG) } & $1^{*}$ & $1^{*}$ & Buccal & $5^{*}$ & $2^{*}$ & $3.5^{\star}$ & $6^{*}$ & $5^{*}$ & $4.5^{*}$ & 1 & 3 \\
\hline & & & Lingual & $4^{*}$ & $2^{*}$ & $4^{*}$ & $5^{*}$ & $7^{*}$ & $5^{*}$ & & 5 \\
\hline \multirow[t]{2}{*}{18 months } & $0^{*}$ & $0^{*}$ & Buccal & $3^{*}$ & $3^{*}$ & $4^{*}$ & $7^{*}$ & $5^{*}$ & $6^{*}$ & $8^{\dagger}$ & $2^{*}$ \\
\hline & & & Lingual & $4^{*}$ & $2^{*}$ & $4^{*}$ & $7^{*}$ & $5^{*}$ & $7^{*}$ & & 3 \\
\hline
\end{tabular}




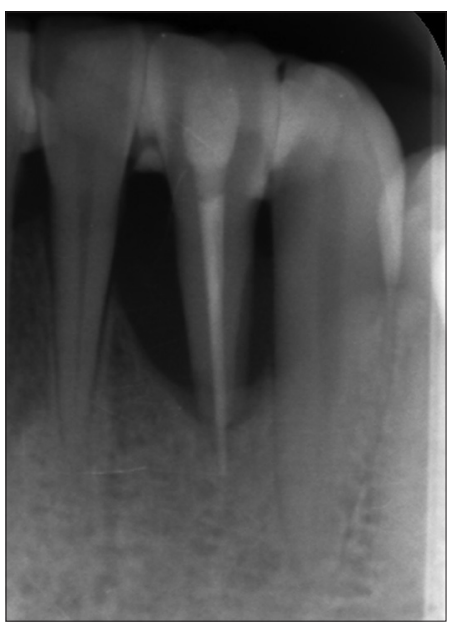

Figure 7: Fifteen months posttreatment periapical radiographic view. Significant bone formation with no external root resorption sign was determined compared to baseline

rich plasma (PRP) in IntR of PHT. Eighteen-month follow-up results of their case demonstrated new bone formation around the root apex. However, no dimensional change was observed in supporting tissues following their procedure. Two study groups combined different regenerative options, including enamel matrix derivatives (EMD), bone grafts, membranes and PRP with IntR to improve tissue support and suggested use of these materials with IntR in order to have more successful and predictable results. ${ }^{[2,4]}$ Baltacioglu et al.$^{[5]}$ evaluated results of IntR of PHT with EMD + demineralized freeze-dried bone allograft (DFDBA) and showed that EMD + DFDBA might develop the outcomes of IntR. Despite comments of these literatures, only periodontal pocket depth and gingival inflammation parameters were evaluated, and no detailed information was given about mucogingival state. To the best of our knowledge, the present case is the first one demonstrating use of FGG to increase periodontal tissue support of a replanted tooth. Following utilization, inflammation symptoms were reduced, and minimal root coverage was obtained with keratinized gingiva enhancement.

\section{CONCLUSION}

In this case-report, 15-month results of mucogingival surgery after IntR were presented. Consequently, treated PHT survived with healthy gingiva, reduced pocket depth, acceptable mucogingival relationship and new hard tissue formation. From the results of this "single case" lack of baseline periapical radiograph and standardized radiographic measurements, IntR supported with FGG may be speculated as an alternative to keep PHT for a period of time. However, randomized controlled follow-up studies are necessary for evidence.

\section{REFERENCES}

1. Demiralp B, Nohutçu RM, Tepe DI, Eratalay K. Intentional replantation for periodontally involved hopeless teeth. Dent Traumatol 2003;19:45-51.

2. Demir B, Demiralp B, Güncü GN, Uyanik MO, Caglayan F. Intentional replantation of a hopeless tooth with the combination of platelet rich plasma, bioactive glass graft material and non-resorbable membrane: A case report. Dent Traumatol 2007;23:190-4.

3. Tözüm TF, Keçeli HG, Serper A, Tuncel B. Intentional replantation for a periodontally involved hopeless incisor by using autologous platelet-rich plasma. Oral Surg Oral Med Oral Pathol Oral Radiol Endod 2006;101:e119-24.

4. Sugai K, Sato S, Suzuki K, Ito K. Intentional reimplantation of a tooth with severe periodontal involvement using enamel matrix derivative in combination with guided tissue regeneration and bone grafting: A case report. Int J Periodontics Restorative Dent 2008;28:89-94.

5. Baltacioglu E, Tasdemir T, Yuva P, Celik D, Sukuroglu E. Intentional replantation of periodontally hopeless teeth using a combination of enamel matrix derivative and demineralized freeze-dried bone allograft. Int J Periodontics Restorative Dent 2011;31:75-81.

6. Silness J, Loe H. Periodontal disease in pregnancy. II. Correlation between oral hygiene and periodontal condtion. Acta Odontol Scand 1964;22:121-35.

7. Loe H, Silness J. Periodontal disease in pregnancy. I. Prevalence and severity. Acta Odontol Scand 1963;21:533-51.

8. Miller PD Jr. Root coverage using a free soft tissue autograft following citric acid application. Part 1: Technique. Int J Periodontics Restorative Dent 1982;2:65-70.

9. Cortellini P, Stalpers G, Mollo A, Tonetti MS. Periodontal regeneration versus extraction and prosthetic replacement of teeth severely compromised by attachment loss to the apex: 5-year results of an ongoing randomized clinical trial. J Clin Periodontol 2011;38:915-24.

\begin{tabular}{|l|l|}
\hline \multicolumn{2}{|c|}{ Access this article online } \\
\hline Quick Response Code: & Website: \\
& www.eurjent.com \\
& \\
\end{tabular}

